\title{
A Novel Gripper design for Diaphragm spring plate Pick and Place Cobot
}

\author{
Jerry Jaison, Vasudevan Nagaraj, Arockia Selvakumar A* \\ Vellore Institute of Technology, Chennai, Tamil Nadu, India \\ *Corresponding author E-mail: arockia.selvakumar@vit.ac.in
}

\begin{abstract}
Robot integrated manufacturing has turned out to be the future of manufacturing automation technology. Worker assisting robots perform simultaneously operation including machining, assembly, inspection, material handling etc. and in some case multiple operations in the same system at a faster and precise rate. Collaborative robots are human and computer controlled hybrid material handling device which facilitates the concept of shared workspace. To exploit its function, gripper mechanisms are inevitable. This project focuses on the design of cobotic grippers for pick and place application. Of the different concepts compared a suitable design is chosen. The gripper moves according to the signals received from the cobot, sensors and PLC control system.
\end{abstract}

Keywords: Cobot; Gripper; Pick and place; Load Checking Machine, Diaphragm Spring Plate.

\section{Introduction}

Nowadays workers are replaced by robots in Original Equipment Manufacturing industries because they provide efficient, advanced and safer means to manufacturing automation. Collaborative robots are robots that are designed to work simultaneously along with human interference. Cabot's move by a unique steering system those are smooth, frictionless and intrinsically stable, cobot particularly appropriate for safety-critical tasks. The cobot's gripper is an end-effector or sometimes called end-of-arm tooling for material handling methods like grasping, holding, moving and controlling materials. We can now see cobot's in occasions where it might be fatal or inaccessible to do specific tasks, for example picking of hazardous or radioactive substances, worst case scenarios like pick and place of explosive bombs where precise motion is mandatory. This transition from manual to automatic system does not reduce job opportunities but enhance the skill set required. Robotic Grippers are very important tools because without it, industrial robot cannot be used in material handling applications. In this project, all aspects with regards to designing a robotic gripper for the purpose of material handling will be discussed.

\section{Background}

There are varieties of diversified classification for grippers according to the end-effector application. Some of the recent models and findings are mentioned below. [1] The design proposed describes about a three fingered mechanically actuated gripper. The gripper is versatile to carry concentric and nonconcentric work piece, by offsetting the position of the finger, with respect to the gripper axis. [2] The paper discusses about the use of universal grippers and how it is useful for gripping objects of different shapes, size and weight. Thus showing how universal grippers and its application for different product types and manufacturing robot application industries. [3] The paper emphasis use of Vacuum grippers in industrial application, its static application.

\section{Methodology}

\subsection{System Model}

The Cobot Gripper is designed for a Diaphragm Load checking machine which checks whether the optimum load according to the specification is attained or not. The material handling operation is changed from manual operation to an automated process. This is achieved with the help of a collaborative robot, a gripper mechanism, a printer to print the load value, one trolley each for loading and Unloading the part. A gripper has to be designed such that it adapts to the auto cycle designed such that the cycle time is reduced. The system layout is shown below:

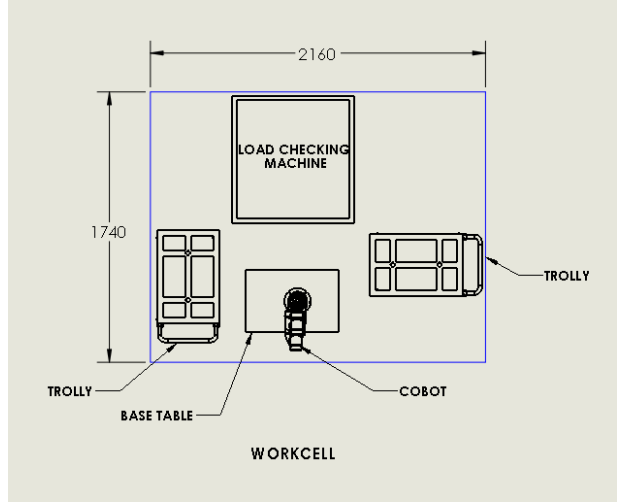

Figure 1: System Work Cell Design 


\subsection{Gripper}

The gripper is designed according to the specification of the cobot. The basic criteria for the gripper is that it maximum payload that it can carry is $5 \mathrm{Kg}$, inclusive of part (Diaphragm Spring plate) weight. The minimum reach required for the gripper $210 \mathrm{~mm}$ due to space constraint. From pre-study it was observed that magnetic grippers are more suitable for ferromagnetic part picking. Thus permanent magnet was chosen over electromagnet to reduce extra power consumption. Another challenge is that the gripper should pick a variety of parts varying from $150 \mathrm{~mm}$ to $180 \mathrm{~mm}$. The various gripper designs were considered and a final design is proposed.

\section{Design Approach and Details}

In the design stage various models are considered and the apt model is subjected to design calculations and manufacturing. Use of multiple gripers are more feasible than the having a single gripper. Thus dual grippers are proposed in all the design shown below. The gripper design is an evolution based design approach. For finalizing the design various parameters are taken into account which will be discussed below:

\subsection{Concept 1}

Proposed model one we use a Neodymium magnet placed in a pot where the magnet is hold tight. There are six cylinders on each gripper plate, of which three are for holding the pot and magnet. The other three are to hold the part during the removal of part form the attractive force of the magnet. In this design the Cobot is attached on one end of the dual gripper arm.

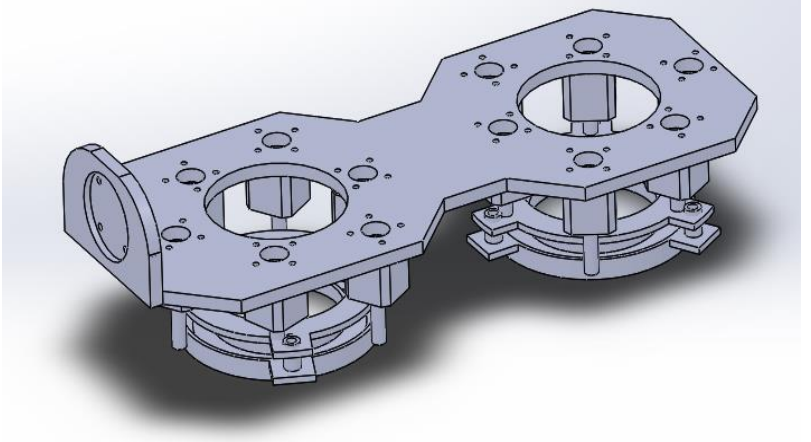

Figure 2: Magnetic Gripper-Model 1

\subsection{Concept 2}

Proposed Model two is similar to model one, the main difference being the position of the position at which the gripper plate is connected with the Cobot. The gripper is connected to the gripper plate in between the two grippers making it double cantilever mechanism. The pick and place is facilitated by the rotation motion of the flange of Cobot. This also facilitates adding more weight at a shorter distance.

\subsection{Concept 3}

Proposed Model three (Angularly Mounted magnetic Grippers) is an innovative design done incorporating magnetic and pneumatic sub-systems together in one system. The design contains a Neodymium magnet placed in a pot for its protection from fatigue, wear and tear. Magnet being a brittle material will break easily after a number of cycles. The magnetization and demagnetization is achieved by motion of this magnet in a cylinder and it's up and down motion is controlled by pneumatic system. This design is a made rectifying almost all the drawbacks of the first design. This is one of reason why the grippers are mounted angularly to make it adjustable to the space constraint.

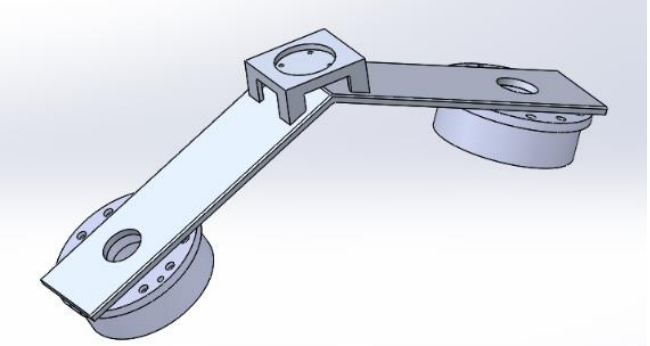

Figure 4: Magnetic Gripper-Model 3

Advantages:

- Robust design.

- Light weight

- Safer convenient than design 1 \& 2

Disadvantages:

- Will cause failure if the lubrication (FRL System) is not proper within the cylinder.

- $\quad$ Contact surface of the part with the gripper should be strong to prevent Frictional wear and tear.

\subsection{Concept Design Comparison}

In this comparison chart various parameter that vary with the designs like stability, weight, reach and accessibility, power requirement. These parameters are compared within the models using a rating method. In this method all the parameters are rated from $1-5$, considering 5 being the best case.

From the design concepts mentioned in Table 1, the most appropriate design is selected as the final design which is model 3.

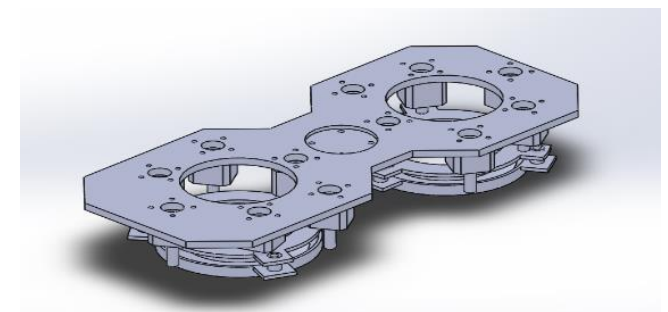

Figure 3: Magnetic Gripper-Model 2 


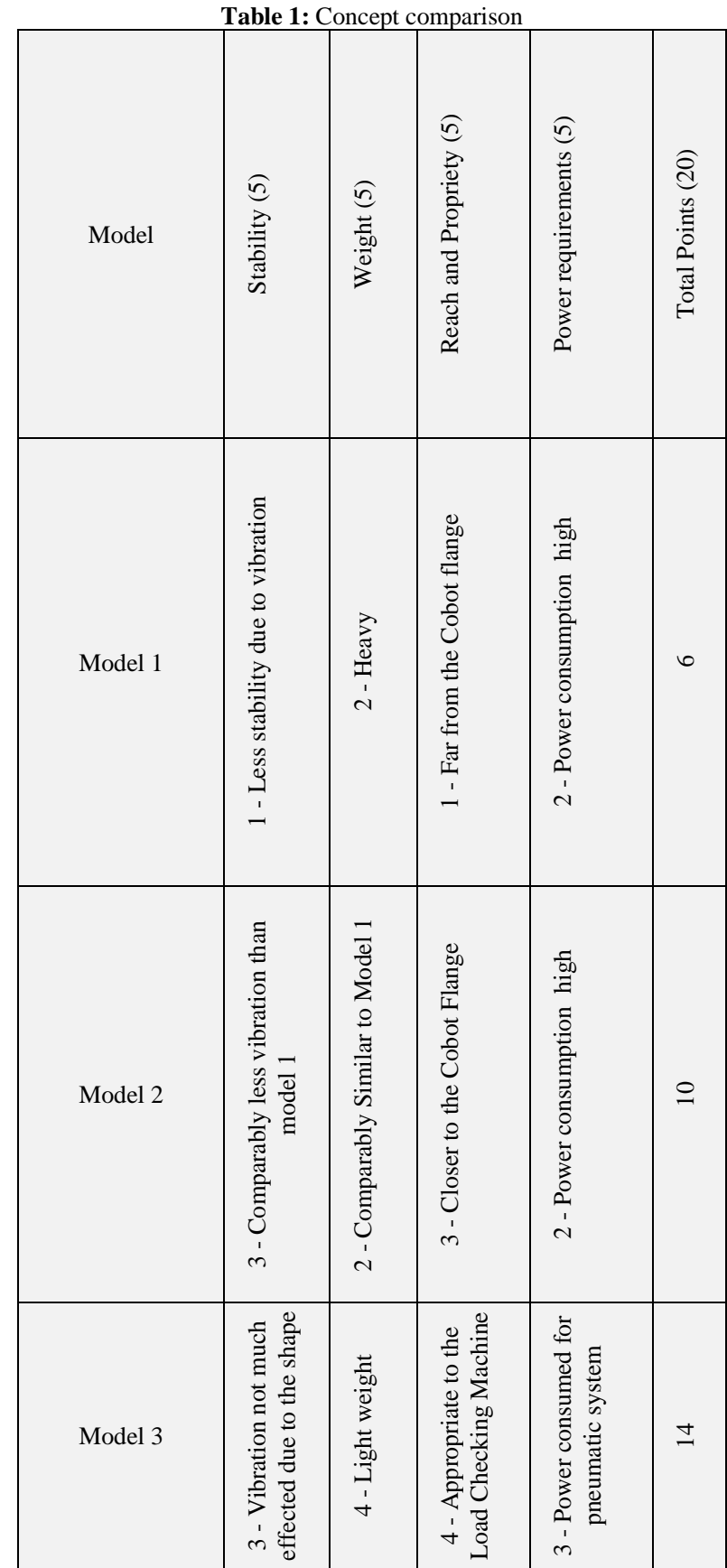

\subsection{Magnet and Pneumatic System Design}

Magnet pull force vs Distance from the center of this magnet were plotted at room temperature:

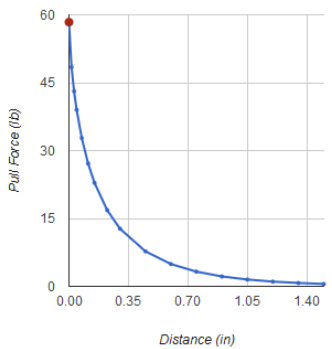

Figure 5: Pull Force vs Distance

From the Graph it can be concluded that maximum magnetic force on the part by the gripper:
BDC position: $71.705332 \mathrm{~N}$

The minimum Stroke length for the cylinder required for the cylinder to drop the part is $14.5 \mathrm{~mm}$.

The minimum pressure required for this piston movement $=$ 0.866205 bar.

\subsection{Design parameters for final gripper}

Table 2 shows the design input parameters of the proposed gripper design.

Table 2: Design Input Parameters

\begin{tabular}{|l|c|}
\hline Weight of Diaphragm Spring Plate & $500 \mathrm{~g}-800 \mathrm{~g}$ \\
\hline Diameter Specification for DSP & $150 \mathrm{~mm}-180 \mathrm{~mm}$ \\
\hline Minimum reach for gripper & $210 \mathrm{~mm}$ \\
\hline Maximum payload for Cobot & $5 \mathrm{Kg}$ \\
\hline Neodymium magnet grade & $\mathrm{N} 35$ \\
\hline
\end{tabular}

The Exploded view of the Gripper assembly is shown in Figure 6.

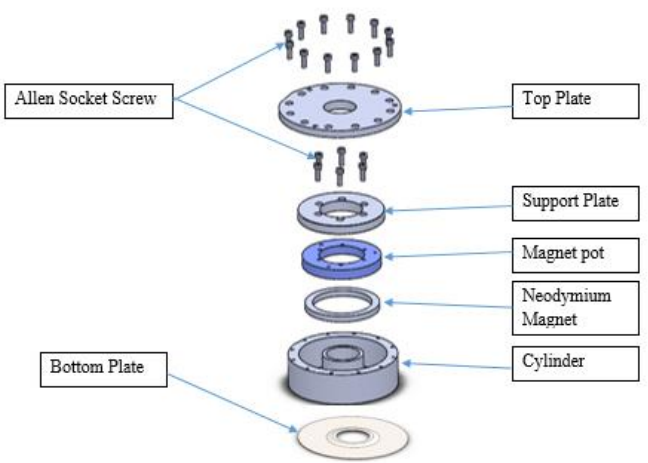

Figure 6: Gripper Exploded View

The sectional view of the gripper is shown in Figure 7.

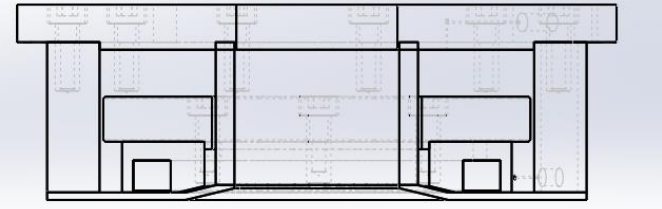

Figure 7: Gripper Sectional view

Strength Simulation was done in Ansys Workbench 16.0. The strength, maximum deformation of the gripper plate was measured. Force calculations were done for the pull force calculations for the Neodymium permanent magnet and the pneumatic pressure calculations system.

\section{Simulation and Analysis}

To check the strength characteristics of the gripper simulation is done in ANSYS Workbench to calculate the maximum stress acting and the deformation characteristics of the angularly mounted gripper. The factors used in the static Analysis are fixed support at the Flange screws, rotational Angular velocity of $\pi$ $\mathrm{rad} / \mathrm{s}$, acceleration due to gravity $9.81 \mathrm{~m} / \mathrm{s}^{2}$. 
The von-mises stress for the gripper plate was found to be less than the maximum permissible stress for that material.

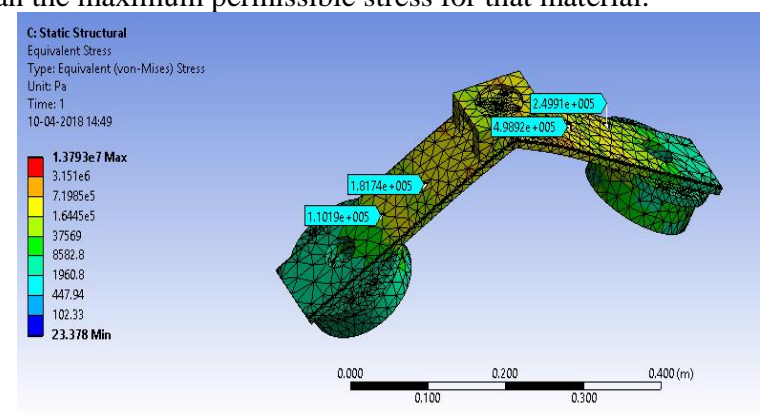

Figure 8: Stress Distribution

Maximum stress $($ actual $)=13.793 \mathrm{MPa}($ Figure $)$

Maximum stress (permissible) $=$ Factor of safety for the gripper plate:

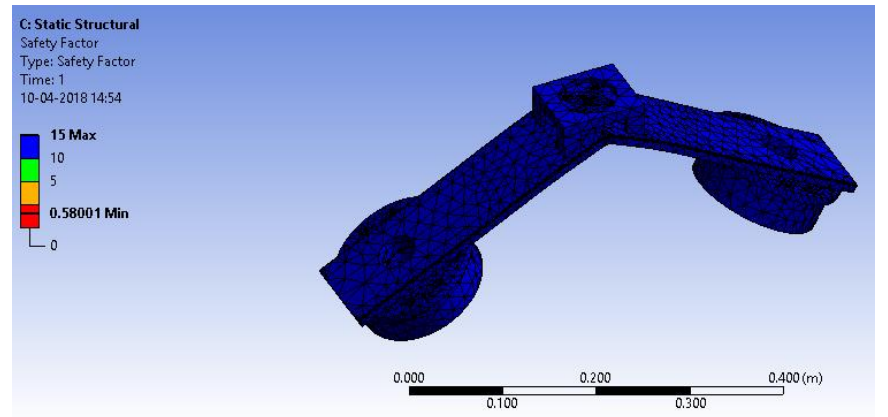

Figure 9: Factor of Safety

\section{Summary}

A novel design of Dual Permanent Magnetic Gripper for a Collaborative Robot is designed and analyzed. Based on the design and analysis, the following findings are summarized:

- The Design of the gripper is done such a way that the use of push rods to pull the part off from the magnet is not required. During the retraction of the piston, part automatically falls on to the Load Checking Machine seating precisely.

- It is observed that he using thin magnets can cause repulsion forces near the center of ring Magnet, due to surface currents. So it is advantages to use long ring magnets.

\section{requirements.}

Use of permanent magnet doesn't require power backup

- Dual Grippers helps to reduce the cycle time of the process by $20 \%$ (Manual cycle Time $=15 \mathrm{~s}$, Automation Cycle time $=12 \mathrm{~s})$

\section{References}

[1] Krishnaraju A, R. R. (2015). Design of Three Fingered RobotGripper Mechanism. International Journal on Mechanical Engineering and Robotics (IJMER), 18-21.

[2] Patakota Venkata Prasad Reddy, V. V. (2013). A Review on Importance of Universal Gripper in Industrial Robot Application. International Journal of Mechanical Engineering and Robotic Research, 1-10.

[3] Patel, S. R. (2015). A Review on Design and Analysis of Pick and Place Robot using Vacuum Gripper. International Journal for Scientific Research \& Development, 1-3. 